\title{
Direct eye contact enhances mirroring of others' movements: A transcranial magnetic stimulation study
}

1

2

\section{Authors and affiliations.}


2 Direct eye contact is a powerful social cue to regulate interpersonal interactions. Previous behavioral studies showed a link between eye contact and motor mimicry, indicating that the automatic mimicry of observed hand movements is significantly enhanced when direct eye contact exists between the observer and the observed model. In the present study, we aim to investigate the neurophysiological basis of the previously reported behavioral enhancements. Here, transcranial magnetic stimulation (TMS) was applied to assess changes in cortico-motor excitability at the level of the primary motor cortex (M1) to explore whether and how the motor system is facilitated from observing others' hand movements and, in particular, how this process is modulated by eye contact. To do so, motor evoked potentials (MEPs) were collected from two hand muscles while participants received single-pulse TMS and naturally observed video clips of an actor showing hand opening movements or static hands. During the observation, either direct or averted eye gaze was established between the subject and the observed actor. Our findings show a clear effect of eye gaze on observation-induced motor facilitation. This indicates that the mapping or 'mirroring' of others' movements is significantly enhanced when movement observation is accompanied by direct eye gaze compared to averted eye gaze. Our results support the notion that eye contact is a powerful social signal with the ability to direct human non-verbal social behavior. Furthermore, our findings are important for understanding the role of the mirror motor system in the mapping of socially relevant actions.

\section{Keywords.}

Movement observation; gaze processing; eye contact; mirror-motor system; transcranial magnetic stimulation 
2 Human social interaction is a complex behavior between two or more individuals to communicate thoughts, intentions, emotional states and actions to one another. Ever since their discovery, 'mirror neurons' have been suggested to form an integral part of the neural circuitry that mediates our capacity to understand the meaning of the actions and behaviors of others (Gallese, 2009).

Neurons with mirror properties were first discovered using single-cell recordings in the ventral premotor cortex of macaque monkeys (Rizzolatti et al., 1988), and were shown to have the ability to fire not only when the monkey executes a certain motor action, but also when the monkey observes another individual performing the motor action (di Pellegrino et al., 1992; Gallese et al., 1996; Rizzolatti et al., 1996). Using movement observation paradigms in combination with functional neuroimaging techniques such as fMRI (Buccino et al., 2001; lacoboni et al., 1999) and PET (Grafton et al., 1996; Rizzolatti et al., 1996) a homologous action observation-execution matching system or 'mirror system' has been localized in the human brain. Particularly, both frontal (inferior frontal gyrus; IFG) and parietal (inferior parietal lobule; IPL) areas have been shown to become increasingly activated during the mere observation of others' actions (Chong et al., 2008; Kilner et al., 2009).

Overall, and according to the notion of 'embodied cognition', this process of 'mapping' observed actions onto the corresponding sensorimotor representations has been hypothesized to form the core neural mechanism by which others' actions and emotional states can be simulated, recognized and understood (lacoboni, 2009; lacoboni et al., 2005; Rizzolatti and Craighero, 2004; Rizzolatti and Fabbri-Destro, 2008). However, note that also weaker accounts of 'embodied cognition' have been put forward, arguing that conceptual 'understanding of actions' may not be represented exclusively in terms of sensorimotor processes, but may additionally involve an abstract or modality-independent representation (Caramazza et al., 2014; Mahon, 2015).

In the past decade, the non-invasive brain stimulation technique transcranial magnetic stimulation (TMS) has been used extensively to measure resonant 'mirror motor' activity in the observer's motor system. By applying TMS over the primary motor cortex (M1), a motor evoked potential (MEP) can be elicited from the contralateral muscles to obtain a measure of corticomotor excitability (Fadiga et al., 1995). Interestingly, a number of studies (for a review, see Fadiga, Craighero and Olivier, 2005) have shown that during the mere observation of others' actions, cortico-motor excitability within parts of M1 becomes increasingly facilitated, as indicated by significant enhancements in MEP amplitudes. Furthermore, this process has been 
shown to be highly muscle-specific, such that modulations in M1 cortico-motor excitability are predominantly observed in the muscles that are used in the observed action (Alaerts, Heremans, Swinnen, \& Wenderoth, 2009; Alaerts, Swinnen, \& Wenderoth, 2009; Strafella \& Paus, 2000). Besides muscular involvement, a number of studies used the TMS technique to explore how different kinematic features of the observed actions are encoded by the observer's motor system, such as temporal dynamics (Gangitano, Mottaghy, \& Pascual-Leone, 2001), grip force (Alaerts, de Beukelaar, Swinnen, \& Wenderoth, 2011; Alaerts et al., 2010; Alaerts, Swinnen, \& Wenderoth, 2010), orientation (Maeda, Kleiner-Fisman, \& Pascual-Leone, 2002) and predictability (Maeda, Chang, Mazziotta, \& lacoboni, 2001).

The mapping mechanism for conveying information from others' behaviors is not only affected by kinematic features, but may also be influenced by the processing of socially relevant cues from the observed environment (Wang and Hamilton, 2012). One such powerful social cue is perceived eye contact. The role of eye gaze in social behavior has been investigated extensively, with several neuroimaging studies showing that observed eye contact is a strong modulator of activity in regions of the 'social brain', a network of structures that is specialized to process social information such as faces, theory of mind and empathy, but also biological motion, action and goal direction (for a review, see Senju and Johnson, 2009). Particularly within the superior temporal sulcus (STS), brain activity has been shown to be specifically enhanced when direct eye contact is perceived (Pageler et al., 2003; Pelphrey, Viola and McCarthy, 2004). Furthermore, the STS region has also been hypothesized to form an integral part of the 'extended' mirror system network by providing the main visual input to upstream fronto-parietal mirror-motor regions (Grèzes et al., 2001; Grossman and Blake, 2002; Grossman et al., 2000).

To date however, only a handful of studies have explored the effect of perceived eye contact on the processing of the actions and movements of others. In terms of movement mimicry, a recent behavioral study by Wang, Newport and Hamilton (2011) provided first indications that the tendency of an observer to mimic others' actions is enhanced when eye contact exists between the observer and the model. As a form of unconscious imitation, mimicry is strongly associated with the mirror neuron system (lacoboni, 2009). In particular, reaction times for mimicking a hand closing or opening movement were shown to be faster when direct eye contact was established, rather than when eye gaze was averted (Wang, Newport, et al., 2011). Also a magnetoencephalographic (MEG) study by Kilner, Marchant and Frith (2006) provided evidence that the social relevance of a stimulus (modulated in terms of the observer's viewpoint) can enhance putative mirror neuron activity. Together, these observations provide 
1 first indications that activity within the human mirror system can be influenced by distinct

2 socially relevant cues from the observed environment.

3

4 To the best of our knowledge, no studies to date have directly investigated the neurophysiological basis of the effect of eye contact on motor resonance, as research has mainly focused on mimicry (Wang, Newport, et al., 2011; Wang, Ramsey, et al., 2011) or the influence of higher-order cognitive processes such as social relevance observation (Kilner et al., 2006). However, since eye contact is a powerful social cue, it would be interesting to directly explore whether direct eye gaze can modulate the mapping of others' actions in the observer's motor system. In the present study, the TMS technique was used to assess the effect of eye gaze on motor facilitation of M1 during movement observation. In particular, single-pulse TMS was applied over left M1 to measure the level of cortico-motor excitability of two hand muscles (right abductor pollicis brevis (APB) and first dorsal interossei (FDI)) during the observation of an actor performing simple hand movements involving those muscles. During the movement observation trials, the actor looked either directly towards or away from the observing participant to assess the effect of direct versus averted eye gaze on observationinduced motor facilitation at the level of M1. If eye gaze forms a salient social cue for modulating the process of mirror-motor mapping at the level of M1, we expected TMS-evoked MEPs to be higher when accompanied by direct gaze compared to averted gaze. 


\section{Material and Methods}

\subsection{Main experiment: Measurements of cortico-motor excitability during movement observation}

\subsubsection{Participants}

Thirty-three right-handed individuals (16 males and 17 females) aged between 19 and 26 years old (mean \pm SD: $22 ; 7 \pm 1 ; 8$ years;months) participated in this study. Handedness was assessed with the Edinburgh Handedness Questionnaire (EHQ; Oldfield, 1971). All participants provided signed written informed consents prior to the experiment, reported no history of neurological/psychiatric illness or motor dysfunctions of the hands/arms and met safety criteria for TMS. Ethical approval for the experiment was granted by the local Ethics Committee for Biomedical Research at the Katholieke Universiteit Leuven and conformed to the Code of Ethics of the World Medical Association (Helsinki, 1964). One female subject was excluded due to technical problems during the experiment.

To explore whether modulating effects of eye gaze were related to inter-individual differences in social responsiveness, subjects completed the Dutch self-report version of the Social Responsiveness Scale for adults (SRS-A; Constantino and Todd, 2005). The SRS-A (64 items) is a widely used screening tool to identify the presence and extent of any social impairments in the typical population using a four-point Likert-scale. It encompasses four subscales, including social awareness (19 items; $\alpha=.80$ ), social communication ( 22 items; $\alpha=.88$ ), social motivation (11 items; $\alpha=.83$ ) and rigidity/repetitiveness (12 items; $\alpha=.79$ ). Lower scores indicate higher social responsiveness. For raw SRS scores, a cut-off point of 54 is suggested for signaling impairments in social responsiveness (Noens et al., 2012).

\subsubsection{General procedure}

Participants were seated in a comfortable chair approximately $80 \mathrm{~cm}$ in front of a widescreen DELL monitor (resolution: $1920 \times 1080$ pixels, refresh frequency: $60 \mathrm{~Hz}$ ) on which video stimuli of hand movements were displayed with a frame rate of $29 \mathrm{~Hz}$. The right hand was placed palm-down on a soft cushion on their lap and participants were asked to relax their hand muscles while spontaneously viewing the presented video clips. During the experiment subjects' vision of their own hands was obstructed by another cushion placed on top of their hands.

\subsubsection{Electromyography recordings and TMS}

Dependent measures of cortico-motor excitability, i.e. motor-evoked potentials (MEPs), were recorded via electromyography (EMG). To do so, disposable self-adhesive electrodes were 
attached to the muscle bellies of the right hand abductor pollicis brevis (APB) and first dorsal interossei (FDI), with two referential electrodes attached at the wrist. Both muscles were shown to be involved in the to-be-observed hand movement (hand opening), although activations were more pronounced for the APB compared to the FDI muscle (see supplementary methods). Single-pulse transcranial magnetic stimulation (TMS), using a Magstim 200 stimulator (Magstim Company Ltd, UK) with a hand-held $70 \mathrm{~mm}$ figure-of-eight coil, was administered to locate the optimal scalp site for stimulating the primary motor cortex (M1) ("hotspotting"). The coil was positioned over the left hemisphere, tangentially to the scalp and $45^{\circ}$ away from the midsagittal line, such that the induced current flow was in a posterior anterior direction, i.e. approximately perpendicular to the central sulcus. Optimal coil location for the experimental TMS-stimulation of M1 was determined as the site that produced maximal responses in the contralateral APB muscle while at rest. Although parameter setting procedures were prioritized for the APB, MEPs were simultaneously obtained from the APB and FDI muscles. Due to the overlap of hand muscle representations in M1, stimulation parameter settings are assumed to be satisfactorily effective for assessing condition-specific modulations simultaneously from both muscles (Facchini et al., 2002; Gertner and Classen, 2006; Krings et al., 1998; Scheiber, 1990). Next, the resting motor threshold (rMT) was defined for each participant as the lowest stimulation intensity that produced a peak-to-peak MEP of at least $50 \mu \mathrm{V}$ in five out of ten consecutive trials (Rossini et al., 1994). During the experimental procedure, stimulation intensity was set at a supra-threshold of $130 \%$ of the subject's rMT (Alaerts, Swinnen and Wenderoth, 2009). Signal Software (version 2.02, Cambridge Electronic Design, UK) was used for EMG-recordings and triggering of the TMS-stimulator. EMG recordings were sampled at $2000 \mathrm{~Hz}$ via a CED Power 1401 unit (Cambridge Electronic Design, UK), amplified, band-pass filtered $(5-1000 \mathrm{~Hz})$ and stored on a PC for offline analysis.

\subsubsection{Video stimuli}

During TMS, video clips were presented to the observing participants. Video stimuli were identical to those used in a previous study by Wang, Newport, et al. (2011) and Wang, Ramsey and Hamilton (2011) in which an actor performed a head movement followed by a simple intransitive (i.e. not directed towards an object) hand movement (figure 1). At the onset of each clip, the actor was facing away from the camera with her eyes closed and her left hand static in front of her face. Then, the actor opened her eyes and turned her head either towards the camera, which resulted in direct gaze towards the observer, or away from the camera, providing averted gaze. Her hand remained static during the duration of the head movement. Subsequently, the actor performed a hand movement (i.e. opening of the hand) or the hand remained static. This resulted in a $2 \times 2$ factorial design with the factors 'observed hand 
movement' (opening or static) and 'gaze direction' (direct or averted). An illustration of the different conditions of the factorial design is provided in figure 1. Each of the four conditions was presented five times in blocks of four five-second video clips (i.e. total of 20 trials per condition). Block presentation order was randomized across subjects and experimental blocks were randomly interleaved with four 'baseline' blocks in which only a blue background was shown. During movement observation, TMS pulses were delivered approximately 4.6 seconds after the start of the video clip which corresponded to the execution phase of the observed hand opening movement (see figure 1). Subjects' attention to the presented videos was randomly assessed between blocks by asking the subject to report the type of hand movement and gaze direction that was previously observed. In $92.5 \%$ of the assessments, subjects gave a correct response, ensuring attention to the presented videos. Video presentation timing was controlled by LabVIEW software (version 14.0, National Instruments, UK) and was triggered by the Signal Software for TMS-stimulation and EMG-recording.

Figure 1. Illustration of the experimental video clips. Participants were presented with a series of video clips of an actor performing a head movement to establish direct or averted gaze towards the observer, followed by a hand opening movement or no movement (static hand). This resulted in a $2 \times 2$ factorial design with the factors 'observed hand movement' (opening or static hand) and 'gaze direction' (direct or averted gaze).

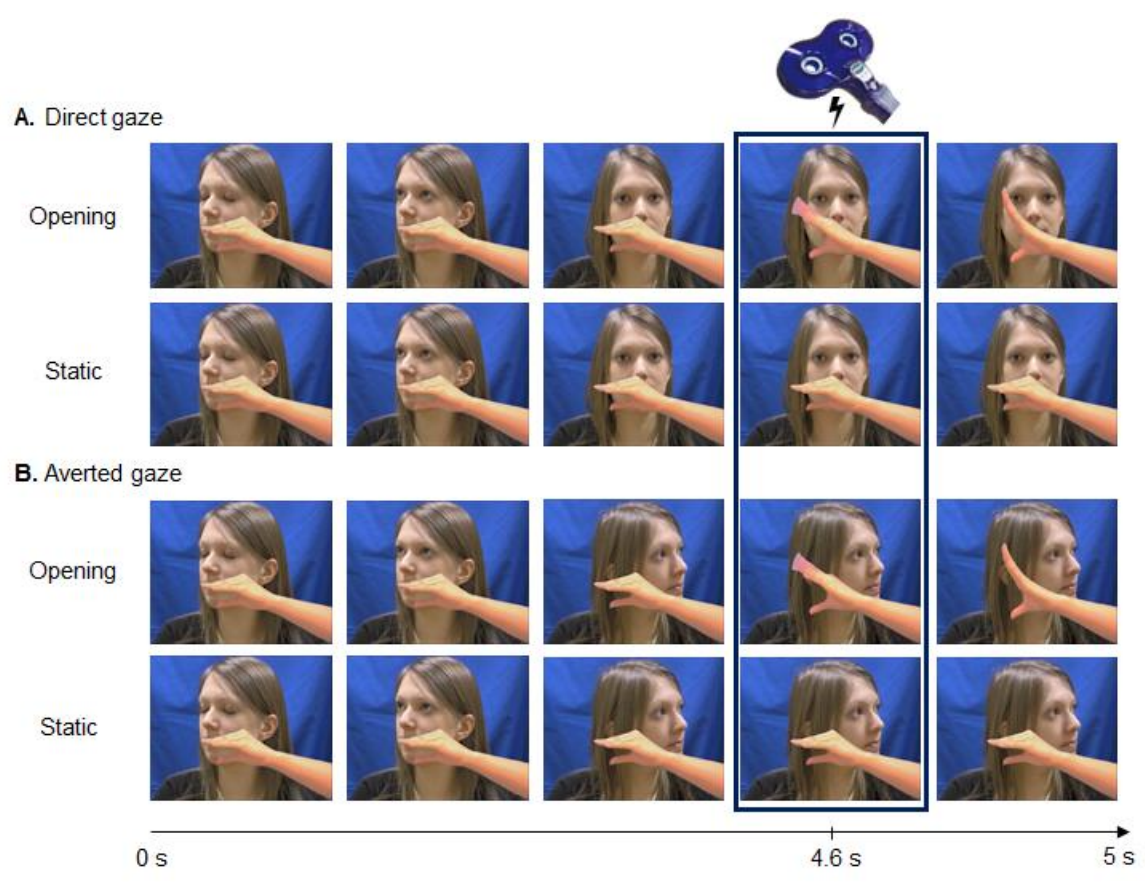

\subsubsection{Data analysis and statistics}

Based on the recorded EMG data, peak-to-peak amplitudes of the TMS-evoked MEPs were determined. Additionally, background EMG was quantified by calculating the root mean square error (RMSE) across the 110 to 10 millisecond interval prior to TMS-stimulation. Since 
background EMG is known to modulate the size of MEP amplitudes (Devanne et al., 1997; Hess et al., 1987), peak-to-peak MEP amplitudes from trials with excessive background EMG (exceeding 2.5 standard deviations away from the mean) were discarded (2.42\% of the trials for the APB, and $1.94 \%$ of the trails for the FDI). Further, MEP peak-to-peak amplitudes were considered as outliers and were removed from the analysis when they exceeded $Q 3 \pm 1.5^{\star}(\mathrm{Q} 3$ Q1), with $Q 1$ and $Q 3$ denoting the first and third quartile computed for each condition in each subject (Electronic Statistics Textbook, 2008, StatSoft). This resulted in an additional omission of $8.48 \%$ of the trials for the APB and $8.45 \%$ of the trails for the FDI. Note that the total number of discarded trials was similar across conditions $(F(4,124)=1.67, p=.16)$ and muscles $(F(1,31)$ $=0.16, p=.70)$.

MEP peak-to-peak amplitudes and RMSE-scores were averaged separately for each condition. Due to high inter-individual differences in raw MEP responses, MEPs recorded during the four experimental conditions were normalized relative to baseline MEP responses separately for each subject (Halaki and Ginn, 2012). Shapiro-Wilk's $W$ tests ensured a normal distribution of the MEP data for each condition. Repeated measures analyses of variance (ANOVAs) with the within-subject factors 'observed hand movement' (opening hand, static hand) and 'gaze direction' (direct gaze, averted gaze) were performed for each muscle (APB, FDI) separately on the normalized MEP amplitudes to explore whether cortico-motor excitability of M1 is modulated by movement observation and/or eye contact. Normalized MEP amplitudes were entered in two repeated measures analyses of variance (ANOVAs), one for each muscle separately, with the within-subjects factors 'observed hand movement' (opening hand, static hand) and 'gaze direction' (direct gaze, averted gaze) to explore whether corticomotor excitability of M1 is modulated by movement observation and/or eye contact. Fisher's least significant difference (LSD) contrasts were used for post-hoc between-condition analyses. All statistics were calculated with Statistica 10 (StatSoft, USA) and results were considered significant with a $p$-value lower than 05 .

\subsection{Control experiment: Gaze behavior during movement observation}

To explore whether gaze behavior and/or attention towards the presented hand movement was similar for the direct and averted eye gaze conditions, an additional eye tracking experiment was conducted while participants observed the four video clips of the main experiment ('observed hand movement' (opening or static) $\times$ 'gaze direction' (direct or averted)). 


\subsubsection{Participants}

Twenty-eight new subjects ( 20 males and 8 females), between the ages of 18 and 29 years participated in the additional eye tracking experiment to measure gaze behavior during observation of the video clips adopted in the main experiment. All participants provided signed written informed consents prior to the experiment and had normal or corrected-to-normal eye vision. Participants of the eye tracking experiment were not the same as those participating in the main movement observation TMS experiment.

\subsubsection{Procedure}

During the eye tracking session, the four video clips as described above were presented on a Tobii T120 binocular eye tracking device. The Tobii eye tracking system consists of a highresolution camera embedded in a 17 inch TFT monitor (resolution: $1280 \times 1024$ pixels, sampling rate: $120 \mathrm{~Hz}$, average precision: $0.5^{\circ}$ of visual angle). Subjects were seated approximately $60 \mathrm{~cm}$ from the device. After a five-point calibration procedure, participants were instructed to naturally view the videos that were shown on the screen. Each of the four conditions was presented in one block of four five-second video clips (i.e. total of four trials per condition). An inter-block interval consisting of a black screen was shown for one second between blocks. The order of block presentation was randomized across subjects.

\subsubsection{Data analysis and statistics}

Two areas of interest (AOI) were defined for each video: the hand region and the eye region. These AOls were defined using rectangular definition tools to mark the corresponding regions. Dependent measures included (i) the total fixation duration (TFD), which measures the sum of the duration for all fixations within an AOI; and (ii) fixation count (FC), which was calculated as the number of times the participant fixates the AOI (i.e. the number of times the participant's eye gaze enters and leaves the AOI). The gaze data was checked for outliers (none) and normality was assessed by means of Shapiro-Wilk's $W$ tests (gaze data was sufficiently normally distributed). For each dependent variable (TFD, FC) and AOI (hand region, eye region), a repeated measures ANOVA with the within-subject factors 'observed hand movement' (opening hand, static hand) and 'gaze direction' (direct gaze, averted gaze) was conducted to examine gaze behavior for each condition. 


\subsection{M1 facilitation during movement observation}

A repeated measures ANOVA with the within-subject factors 'observed hand movement' (opening hand, static hand) and 'gaze direction' (direct gaze, averted gaze) was conducted on the normalized MEP data separately for each muscle (APB, FDI) to explore the effect of eye gaze on observation-induced facilitation of the primary motor cortex (M1). Figure 2 displays the MEP amplitude data separately for each muscle and condition.

In both muscles, a two-way interaction between 'observed hand movement' and 'gaze direction' was revealed (tentatively in the APB: $F(1,31)=2.89 ; p=0.06 ; \eta^{2}=.09$; power $=.38$; significantly in the FDI: $F(1,31)=7.07 ; p<.05, \eta^{2}=.19$; power $\left.=.73\right)$, indicating a differential impact of eye gaze on observation-induced M1 facilitation. Direct exploration of the difference in MEP response between direct and averted eye gaze showed that for observing the hand opening movement, MEP responses of the APB and FDI were significantly higher for the direct eye gaze condition compared to the averted eye gaze condition (Fisher LSD: both $p<.05$; figure 2, left panel). During observation of the static hand condition, MEP responses in the APB muscle were not significantly different between the direct and averted eye gaze conditions $(p=.75)$, whereas in the FDI muscle, M1 facilitation was reversibly modulated, indicating significantly lower MEP responses when the static hand observation was accompanied by direct versus averted eye gaze $(p<.001$; figure 2 , right panel). No main effects of 'gaze direction' (all $p>.29$ ) or 'observed hand movement' (all $p>.48$ ) were revealed in either muscle.

MEP scores were not confounded by modulations in background EMG scores. This was tested by conducting similar ANOVAs to the corresponding background EMG data (i.e. normalized RMSE-scores). Background EMG was generally small and condition-specific modulations were minimal, as no significant main or interaction effects were revealed. For all conditions and muscles, background EMG scores are listed in supplementary table 1. 
Figure 2. Normalized MEP peak-to-peak amplitude data for the APB and FDI during action observation, separately for each condition. Vertical bars denote standard errors of the mean. * Denotes a significant difference between conditions $(p<.05)$.

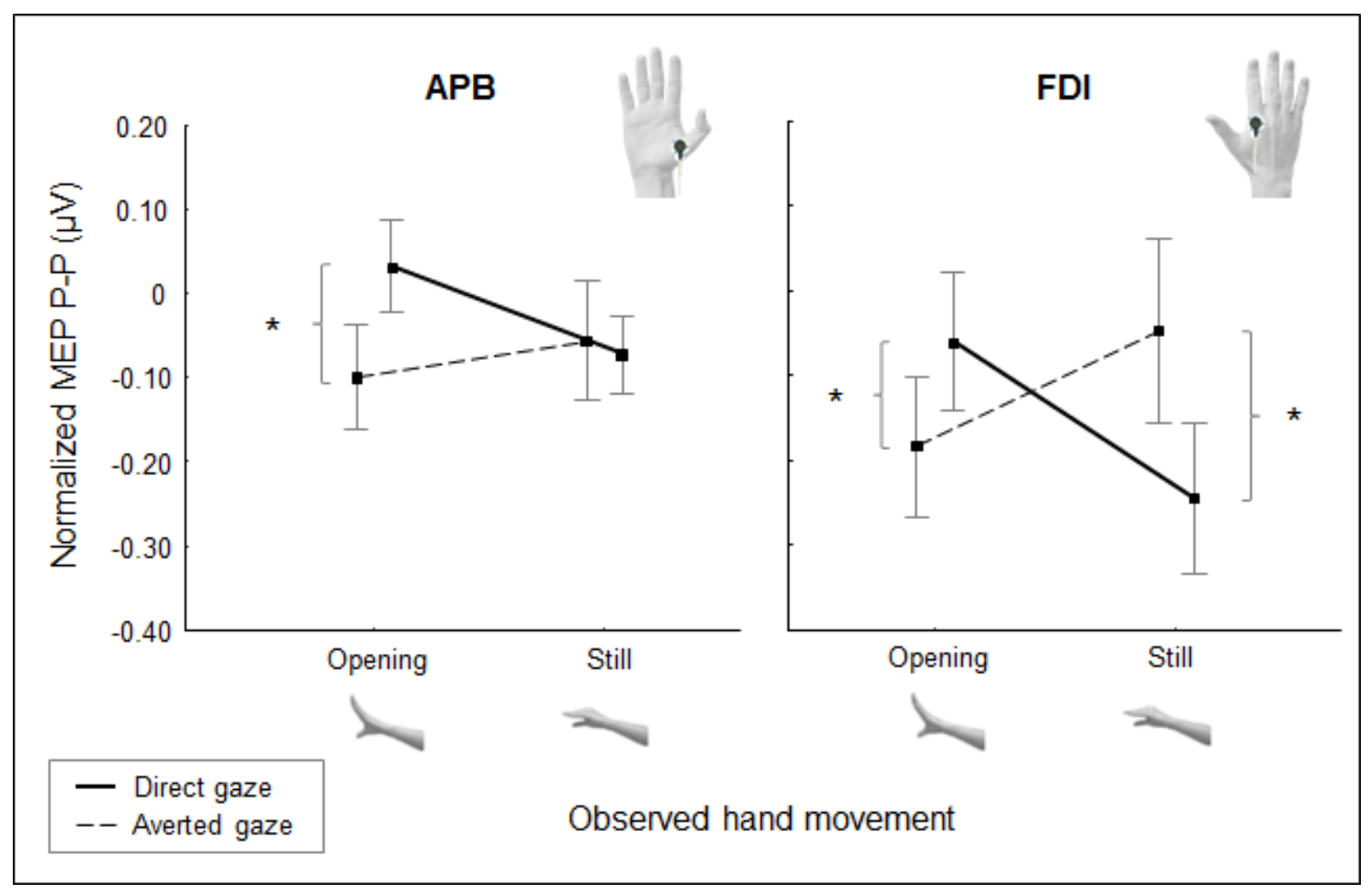

\subsection{Link with social responsiveness}

Participants reported a mean total SRS-A score of 36.15 (SD =19.74), which, as a group, is well below the cut-off score of 54 signaling impairments in social responsiveness. However, for five out of the 32 participants a total score higher than the cut-off point was reported, indicative of impairments in terms of social responsiveness. To explore whether inter-individual differences in social responsiveness were related to the extent by which direct gaze elicited higher MEP responses compared to averted gaze during movement observation, a regression analysis was conducted with 'gaze effect' (difference in MEP response between direct and averted eye gaze conditions) as dependent variable and 'social responsiveness (sub)score' as predictor (across muscles). Overall, beta-values were generally small and none of the relationships reached significance (all $p>.25$, see supplementary table 2 ) indicating that social responsiveness was not predictive for the extent of M1 facilitation.

\subsection{Gaze behavior during movement observation}

An additional eye tracking experiment was conducted to explore whether the observer's gaze behavior was different when the movement observation conditions (opening hand, static hand) were accompanied by direct versus averted gaze. To do so, eye tracking was performed and 
1 the total fixation duration (TFD) and fixation count (FC) were determined in an area-of-interest

2 (AOI) centered over the hand and eye region of the presented video clip.

For the TFD, a repeated-measures ANOVA with the within-subject factors 'observed hand movement' (opening hand, static hand) and 'gaze direction' (direct, averted) revealed no significant main effect of 'gaze direction', indicating that across movement observation conditions, participants fixated an equal amount of time towards the hand region during direct as during averted gaze conditions $\left(F(1,27)=0.79, p=.38, \eta^{2}=.03\right.$, power $\left.=.14\right)$. Similarly, no significant effect of 'gaze direction' was revealed for the FC data, indicating that participants made a comparable amount of saccades towards the hand region for the direct and averted gaze conditions $\left(F(1,27)=0.75, p=.39, \eta^{2}=.03\right.$, power $\left.=.13\right)$. Note however, that the ANOVA revealed significant main effects of 'observed hand movement', indicating that irrespective of gaze direction (direct, averted), subjects looked significantly more (FC: $F(1,27)=21.24, p<$ $.0001, \eta^{2}=.44$, power $\left.=.99\right)$ and longer (TFD: $F(1,27)=26.62, p<.0001, \eta^{2}=.50$; power $=$ .99) towards the hand region when the actor performed the opening hand movement, compared to when the actor's hand remained static.

The eye tracking data were additionally used to explore whether direct versus averted eye gaze differentially modulated the observer's gaze behavior towards the eye region of the actor. Not surprisingly, for the eye region AOI, a significant main effect of 'gaze direction' was revealed, indicating that across hand movements, subjects looked longer towards the eye region during direct gaze than during averted gaze (i.e. indicative of the establishment of eye contact between the actor and the observer during direct gaze conditions) (TFD: $F(1,27)=$ $8.73, p<.01, \eta^{2}=.24$; power $=.81$ ). In terms of FC, participants made a comparable amount of saccades toward the eye region during direct gaze as during averted gaze $(F(1,27)=0.06$, $p=.81, \eta^{2}=.002$, power $=.06$ ). Mean TFD and FC values are displayed separately for each $\mathrm{AOI}$ and condition in supplementary table 3. 
2 In the present study, transcranial magnetic stimulation (TMS) was used to assess corticomotor excitability at the level of the primary motor cortex (M1) during movement observation, and, in particular, whether observation-induced facilitation of $\mathrm{M} 1$ is altered when accompanied by direct or averted eye gaze. Overall, our results show that observation-induced M1 facilitation was most pronounced when direct eye gaze was observed, indicating that eye gaze forms a salient social cue that can modulate the extent by which others' actions are 'mapped' onto the observer's motor system.

As such, the present TMS study extends previous findings from behavioral studies by Wang, Newport, et al. (2011) and Wang, Ramsey, et al. (2011) studying the effect of eye gaze on automatic motor mimicry using similar video clips as those adopted in the present study. In these studies, a stimulus-response compatibility paradigm was adopted where participants were asked to perform the same movement or the opposite movement as viewed in the video clip, and a clear congruency effect was found indicating that responses were significantly faster when the same movement was performed (e.g. hand opening observed - hand opening performed), compared to trials in which the opposite hand movement was performed (e.g. hand closing observed - hand opening performed). Interestingly, Wang, Newport, et al. (2011) demonstrated that this mimicry congruency effect was even more enlarged when direct eye contact was established between the observer and the observed actor, indicating a rapid modulation of mimicry by eye contact. Our study provides insights into the neurophysiological mechanism underlying this modulating effect of eye gaze on automatic motor simulation, by showing that direct eye gaze can significantly enhance the extent by which the observed movement is mirrored onto the observer's motor system. Our findings are also in agreement with previous results from an MEG study by Kilner et al. (2006). In this study, MEG was used to record cortico-motor activity whilst participants observed upper limb movements of an actor that was facing away or towards them. Results from this study showed that cortico-motor responses to movement observation (oscillatory activity in the $7-12 \mathrm{~Hz}$ frequency range) are dependent on the relative perspective of the observed model towards the observer, such that cortico-motor modulations to movement observation were only present when the actor was facing towards the observer, not when the actor was facing away from the observer. The authors suggested that distinct 'social' signals accompanying the observed movements and actions of other people (e.g. the perspective relative to the observer) can modulate visuospatial attention, such that only specific visual information of the most salient and most socially relevant actions is allowed to enter the mirror system for further processing. Our study extends these findings by showing that not the perspective per se, but the establishment of direct eye gaze may be a highly salient cue in determining the extent by which an observed action will be 
mapped onto the observer's motor system. In addition to perceived eye contact, also other social signals may sophistically direct motor resonance (Wang and Hamilton, 2012). Indeed, previous studies have shown that also social cues such as self-construal (Obhi et al., 2011), social interaction (Hogeveen and Obhi, 2012) and power (Hogeveen et al., 2014) can influence motor resonance. It has therefore been argued that the control of motor resonance may involve a 'social top-down response modulation' (STORM) that is dependent on the social context in which others' actions are observed (Wang and Hamilton, 2012).

Previous fMRI studies in humans (Kampe et al., 2003; Nummenmaa and Calder, 2009; Senju and Johnson, 2009b) and single-cell recordings in monkeys (Emery, 2000; Perrett et al., 1992) consistently showed that the medial prefrontal cortex (mPFC) and the superior temporal sulcus (STS) are increasingly activated during direct versus averted gaze, highlighting the importance of these two regions in gaze processing. Wang, Ramsey, et al. (2011) replicated these findings and additionally suggested that the MPFC may well be the originator of the effect of eye gaze on motor mimicry by modulating functional connectivity with the STS, i.e. the main visual input region to the fronto-parietal mirror system. In other words, the model by Wang, Ramsey, et al. (2011) suggested that gaze-related activations at the level of mPFC may impose a top-down control over the processing of visuo-motor information at the level of the STS, which in turn may impact the extent by which observed actions are processed in down-stream mirror regions in the inferior frontal gyrus, ventral premotor regions and inferior parietal lobule. In this view, M1 may be conceived as the end-state region of a chain of cortico-cortical connections signaling on whether or not the cortico-spinal tract and the corresponding peripheral muscles are to be recruited for initiating overt motor simulation. Correspondingly, by receiving direct input from upstream premotor and mirror regions, modulations in cortico-motor excitability at the level of M1 may reflect an end-state cortical measure of how the brain 'evaluated' the social relevance or saliency of the observed visual scene. Our results therefore provide additional support to the notion that direct eye gaze from the actor forms a strong mediator for evaluating whether or not visuo-motor information of the observed action is sufficiently relevant to be processed up to the level of M1. Instead of simulating all possible movement-related information perceived in a visual scene, eye contact may direct the motor system to give preference to processing visuo-motor input originating from the most socially relevant person. Note that in the FDI muscle, but not in the APB, we found an inverse effect of eye gaze on M1 facilitation during the observation of the static hand, indicating increased M1 excitability for the averted compared to the direct gaze condition. One potential interpretation could be that during the trials in which no actual hand opening movement was observed (only a static hand), direct eye gaze might have induced an increased inhibitory effect on M1 excitability to encode more efficiently that no movement is observed during these trials. From this perspective, it can be 
1 hypothesized that direct eye primes the observation-to-execution mapping system by

2 increasing the signal-to-noise ratio when perceiving motion stimuli (i.e., by effectively heightening M1 excitability during actual movement observation, and inhibiting M1 excitability when no movement is observed). This interpretation remains speculative however as it is unclear why this effect was then only significantly observed in the FDI muscle and not in the APB muscle.

Nummenmaa and Calder (2009) showed that observing another person's gaze can automatically induce gaze following, thereby shifting spatial attention toward the scene observed by the model. In this view, an alternative explanation for the observed gaze effect of the present study can be put forward, suggesting that the averted gaze conditions induced a shift of spatial attention away from the observed hand movement, thereby reducing the observation-induced M1 facilitation. We explicitly addressed this alternative explanation in a control eye-tracking experiment, in which the same video clips were displayed while the participants' gaze behavior was recorded. Overall, we found no indications that participants spend less time fixating on the to-be-observed hand movement in video clips with averted gaze compared to direct gaze, which makes it implausible that differences in visual spatial attention are responsible for the encountered gaze-related modulations in observation-induced M1 excitability. Also Wang, Newport, et al. (2011) explicitly addressed this issue in their control "flashbox" experiment in which distracting stimuli (i.e. flashing white squares) were displayed in the periphery during the movie clip to draw participant's attention. These manipulations did however not alter the effects of eye gaze on motor mimicry, indicating the robustness of the eye gaze effect relative to the presence of distracters competing for attention. Furthermore, previous studies have shown that eye gaze can have a direct effect on several physiological measures such as skin conductance, indicating a heightened response of the observer's autonomic nervous system during direct versus averted eye gaze (Hietanen et al., 2008; Pönkänen et al., 2011). While direct eye gaze conditions may have induced a similar heightening of arousal in the present study, it is unlikely that these arousal effects directly affected the modulation of MEP responses, since enhancements in M1 facilitation were specifically observed during the movement observation condition (i.e. hand opening) and not during the observation of the static hand. Also background EMG scores - which were measured before the TMS pulse - did not show any condition specific modulations.

Nevertheless, future research on the effect of eye gaze on motor facilitation would benefit from the inclusion of parallel assessments of skin conductance to explore the possibility of arousalrelated effects further. Also in terms of the assessment of viewing behavior and attention to the presented stimuli, future studies would benefit from the inclusion of online eye tracking. In 
the present study, assessments of viewing behavior were only assessed in a separate eye tracking experiment in which the participants were different from those participating in the main experiment. While this additional eye tracking experiment already provided relevant information related to viewing behavior towards the presented stimuli, online eye tracking would have allowed a direct assessment of viewing behavior and attention on a trial-by-trial basis and its potential relationship to the evoked MEP responses. In the present TMS experiment, attention to the presented stimuli was only assessed randomly, by asking the participants to verbally report the type of hand movement and gaze direction that was observed in the previous video clip. Since this assessment might have affected the subjects' explicit awareness of the presented experimental manipulations, it should be beneficial for future experiments to adopt other strategies to assess the subjects' online attention to the presented stimuli, such as the inclusion of eye tracking.

Overall, mirror motor mapping is hypothesized to form the basic neural mechanism by which others' actions and emotional states can be simulated, recognized and imitated. Considering the hypothesized link between motor simulation and these high-level social skills, the 'broken mirror theory of autism' has been put forward, postulating that behavioral deficits in action understanding, imitation and empathy seen in Autism Spectrum Disorders (ASD) may result from aberrant functioning of the fronto-parietal mirror motor mapping (Ramachandran and Oberman, 2016). While a number of neurophysiological studies provided support for aberrant 'mirroring' in ASD (Dapretto et al., 2006; Enticott et al., 2012; Hadjikhani et al., 2006; Oberman et al., 2005), also several studies found no evidence to support this account (for a review, see Hamilton, 2013). Related to these controversies, and considering that eye contact may form a highly salient cue and perhaps even a prerequisite for the initiation of motor simulation, it would be interesting for future research to evaluate whether and how gaze-related effects on motor mirroring are affected in patients with ASD, who are well-known to display particular difficulties with engaging mutual eye contact (Kaartinen et al., 2012; Kylliäinen and Hietanen, 2006; Kylliäinen et al., 2012; Senju and Johnson, 2009a). Note that in the present study, we found no significant relationships between scores on the social responsiveness scale (SRS) and the extent of the 'gaze effect'. However, considering that inter-individual differences in SRS scores were generally small in our rather homogenous sample of neurotypical students, it should be interesting for future studies to explore the relationship between the eye gaze effect and social responsiveness in more heterogeneous samples including individuals with particular implications in the social domain, such as ASD or social anxiety disorders (Myllyneva et al., 2015; Wieser et al., 2009). 
1 Further, considering that based on the present sample, uncertainty exists with regard to the

2 behavioral correlates of the observed gaze effect on motor mirroring, it should be interesting for future studies to explore whether - in addition to social competence - potentially also other personality traits may be informative in predicting inter-individual variations in the observed gaze effect. For example, based on the work by Hietanen et al. (2008), several links have been suggested between seeing direct or averted eye gaze and inter-individual differences in the motivational system towards approach and avoidance. In particular, neuroticism and scales assessing social phobia have been suggested to form important predictors of behavioral direct gaze avoidance and subjective averted gaze preference (Myllyneva et al., 2015; Uusberg et al., 2015). In this view, it should also be interesting for future research to discern whether interindividual differences in these motivational preferences towards eye contact may be important in determining the facilitative effect of eye gaze on motor mirroring.

To sum up, the present results provide evidence that the mapping of others' movements onto the observer's motor system is enhanced when direct compared to averted eye gaze is established between the observer and the observed model. These findings support the notion that eye contact is a powerful and highly salient social signal with the ability to modify activity in the human mirror-motor system, thereby directing human social interactions.

\section{$5 \quad$ Funding}

This research was supported by grants from the Flanders Fund for Scientific Research (FWO [KAN 1506716N, KAN 1521313N and G.0401.12] and the Branco Weiss fellowship of the Society in Science - ETH Zurich granted to K.A. J.P. is supported by an internal fund of the KU Leuven [STG/14/001].

\section{Acknowledgments}

The authors would like to thank Jaana van Overwalle, Michaël Doumen and Stephanie Brams for their assistance in data collection and analysis. We would also like to thank Prof. dr. Antonia Hamilton for her contribution in inspiring the design of the present study.

\section{Abbreviations}

APB, abductor pollicis brevis; FC, fixation count; FDI, first dorsal interosseous; IFG, inferior frontal gyrus; IPL, inferior parietal lobule; M1, primary motor cortex; MEP, motor-evoked 
1 potential; mPFC, medial prefrontal cortex; RMSE, root mean square error; rMT, resting motor 2 threshold; STS, superior temporal sulcus; TFD, total fixation duration; TMS, transcranial 3 magnetic stimulation. 


\section{References}

Alaerts, K., de Beukelaar, T. T., Swinnen, S. P., \& Wenderoth, N. (2012). Observing how others lift light or heavy objects: Time-dependent encoding of grip force in the primary motor cortex. Psychological Research, 76(4), 503-531. doi:10.1007/s00426-011-0380-1

Alaerts, K., Heremans, E., Swinnen, S. P., \& Wenderoth, N. (2009). How are observed actions mapped to the observer's motor system? Influence of posture and perspective. Neuropsychologia, 47(2), 415-422. doi:10.1016/j.neuropsychologia.2008.09.012

Alaerts, K., Senot, P., Swinnen, S. P., Craighero, L., Wenderoth, N., \& Fadiga, L. (2010). Force requirements of observed object lifting are encoded by the observer's motor system: $A$ TMS study. European Journal of Neuroscience, 31(6), 1144-1153. doi:10.1111/j.14609568.2010.07124.x

Alaerts, K., Swinnen, S. P., \& Wenderoth, N. (2009). Is the human primary motor cortex activated by muscular or direction-dependent features of observed movements? Cortex, 45(10), 1148-1155. doi:10.1016/j.cortex.2008.10.005

Alaerts, K., Swinnen, S. P., \& Wenderoth, N. (2010). Observing how others lift light or heavy objects: Which visual cues mediate the encoding of muscular force in the primary motor cortex? Neuropsychologia, 48(7),

2082-2090. doi:10.1016/j.neuropsychologia.2010.03.029

Buccino, G., Binkofski, F., Fink, G. R., Fadiga, L., Fogassi, L., Gallese, V., ... Freund, H.-J. (2001). Action observation activates premotor and parietal areas in a somatotopic manner: An fMRI study. European Journal of Neuroscience, 13(2), 400-404. doi:10.1111/j.1460-9568.2001.01385.x

Caramazza, A., Anzellotti, S., Strnad, L., \& Lingau, A. (2014). Embodied cognition and mirror neurons: A critical assessment. Annual Review of Neuroscience, 37, 1-15. doi: 10.1146/annurev-neuro-071013-013950.

Chong, T. T.-J., Cunnington, R., Williams, M. A., Kanwisher, N., \& Mattingley, J. B. (2008). $\mathrm{fMRI}$ adaptation reveals mirror neurons in human inferior parietal cortex. Current Biology, 18(20), 1576-1580. doi:10.1016/j.cub.2008.08.068

Constantino, J. N., \& Todd, R. D. (2005). Intergenerational transmission of subthreshold autistic traits in the general population. Biological Psychiatry, 57(6), 655-660. doi:10.1016/j.biopsych.2004.12.014

Dapretto, M., Davies, M. S., Pfeifer, J. H., Scott, A. A., Sigman, M., Bookheimer, S. Y., \& lacoboni, M. (2006). Understanding emotions in others: mirror neuron dysfunction in children with autism spectrum disorders. Nature Neuroscience, 9, 28-30. doi:10.1038/nn1611

de Beukelaar, T. T., Alaerts, K., Swinnen, S. P., \& Wenderoth, N. (2015). Motor facilitation during action observation: The role of M1 and PMv in grasp predictions. Cortex, 75, 180 
192. doi:10.1016/j.cortex.2015.11.009

Devanne, H., Lavoie, B. A., \& Capaday, C. (1997). Input-output properties and gain changes in the human corticospinal pathway. Experimental Brain Research, 114(2), 329-338.

di Pellegrino, G., Fadiga, L., Fogassi, L., Gallese, V., \& Rizzolatti, G. (1992). Understanding motor events: A neurophysiological study. Experimental Brain Research, 91(1), 176-180. doi:10.1007/BF00230027

Emery, N. J. (2000). The eyes have it: the neuroethology, function and evolution of social gaze. Neuroscience and Biobehavioral Reviews, 24(6), 581-604. doi:10.1016/S01497634(00)00025-7

Enticott, P. G., Kennedy, H. A., Rinehart, N. J., Tonge, B. J., Bradshaw, J. L., Taffe, J. R., Daskalakis, Z. J., \& Fitzgerald, P. B. (2012). Mirror neuron activity associated with social impairments but not age in autism spectrum disorder. Biological Psychiatry, 71, 427-33. doi:10.1016/j.biopsych.2011.09.001

Facchini, S., Romani, M., Tinazzi, M., \& Agliotti, S. M. (2002). Time-related changes of excitability of the human motor system contingent upon immobilisation of the ring and little fingers. Clinical Neurophysiology, 113(3), 367-375.

Fadiga, L., Craighero, L., \& Olivier, E. (2005). Human motor cortex excitability during the perception of others' action. Current Opinion in Neurobiology, 15(2), 213-218. doi:10.1016/j.conb.2005.03.013

Fadiga, L., Fogassi, L., Pavesi, G., \& Rizzolatti, G. (1995). Motor facilitation during action observation: a magnetic stimulation study. Journal of Neurophysiology, 73(6), 26082611.

Gallese, V. (2009). Motor abstraction: A neuroscientific account of how action goals and intentions are mapped and understood. Psychological Research, 73(4), 486-498.

Gallese, V., Fadiga, L., Fogassi, L., \& Rizzolatti, G. (1996). Action recognition in the premotor cortex. Brain, 119(2), 593-609. doi:10.1093/brain/119.2.593

Gangitano, M., Mottaghy, F. M., \& Pascual-Leone, A. (2001). Phase-specific modulation of cortical motor output during movement observation. NeuroReport, 12(7), 1489-1492.

Gertner, R., \& Classen, J. (2006). Modular organization of finger movements by the human central nervous system. Neuron, 52(4), 731-742.

Grafton, S. T., Arbib, M. A., Fadiga, L., \& Rizzolatti, G. (1996). Localization of grasp representations in humans by positron emission tomography. Experimental Brain Research, 112(1), 103-111. doi:10.1007/BF00227183

Grèzes, J., Fonlupt, P., Bertenthal, B., Delon-Martin, C., Segebarth, C., \& Decety, J. (2001). Does perception of biological motion rely on specific brain regions? Neurolmage, 13(5), 775-785. doi:10.1006/nimg.2000.0740

Grossman, E. D., \& Blake, R. (2002). Brain areas active during visual perception of biological 
motion. Neuron, 35(6), 1167-1175. doi:10.1016/S0896-6273(02)00897-8

Grossman, E., Donnelly, M., Price, R., Pickens, D., Morgan, V., Neighbor, G., \& Blake, R. (2000). Brain areas involved in perception of biological motion. Journal of Cognitive Neuroscience, 12(5), 711-720. doi:10.1162/089892900562417

Hadjikhani, N., Joseph, R. M., Snyder, J., \& Tager-Flusberg, H. (2006). Anatomical differences in the mirror neuron system and social cognition network in autism. Cerebral Cortex, 16, 1276-1282. doi:10.1093/cercor/bhj069

Halaki, M., \& Ginn, K. (2012). Normalization of EMG signals: To normalize or not to normalize and what to normalize to? In G.R. Naik (Ed.), Computational Intelligence in Electromyography Analysis - A Perspective on Current Applications and Future Challenges (pp. 175-194). InTech. doi: 10.5772/49957

Hamilton, A.F. de C. (2013). Reflecting on the mirror neuron system in autism: A systematic review of current theories. Developmental Cognitive Neuroscience, 3, 91-105. doi:10.1016/j.dcn.2012.09.008

Hess, C. W., Mills, K. R., \& Murray, N. M. (1987). Responses in small hand muscles from magnetic stimulation of the human brain. Journal of Physiology, 388, 397-419.

Hietanen, J. K., Leppänen, J. M., Peltola, M. J., Linna-Aho, K., \& Ruuhiala, H. J. (2008). Seeing direct and averted gaze activates the approach-avoidance motivational brain systems. Neuropsychologia, 46(9), 2423-2430. doi:10.1016/j.neuropsychologia.2008.02.029

Hogeveen, J., Inzlicht, M., \& Obhi, S. S. (2014). Power changes how the brain responds to others. Journal of Experimental Psychology, 143, 755-762.

Hogeveen, J., \& Obhi, S. S. (2012). Social interaction enhances motor resonance for observed human actions. The Journal of Neuroscience, 32(17), 5984-5989. doi:10.1523/JNEUROSCI.5938-11.2012

lacoboni, M. (2009). Imitation, empathy, and mirror neurons. Annual Review of Psychology, 60, 653-70. doi:10.1146/annurev.psych.60.110707.163604

lacoboni, M., Molnar-Szakacs, I., Gallese, V., Buccino, G., Mazziotta, J. C., \& Rizzolatti, G. (2005). Grasping the intentions of others with one's own mirror neuron system. PLoS Biology, 3(3), e79. doi:10.1371/journal.pbio.0030079

lacoboni, M., Woods, R. P., Brass, M., Bekkering, H., Mazziotta, J. C., \& Rizzolatti, G. (1999). Cortical mechanisms of human imitation. Science, 286(5449), 2526-2528. doi:10.1126/science.286.5449.2526

Kaartinen, M., Puura, K., Mäkelä, T., Rannisto, M., Lemponen, R., Helminen, M., ... Hietanen, J. K. (2012). Autonomic arousal to direct gaze correlates with social impairments among children with ASD. Journal of Autism and Developmental Disorders, 42(9), 1917-1927. doi:10.1007/s10803-011-1435-2

Kampe, K. K. W., Frith, C. D., \& Frith, U. (2003). "Hey John”: Signals conveying communicative 
intention toward the self activate brain regions associated with "mentalizing", regardless of modality. Journal of Neuroscience, 23(12), 5258-5263.

Kilner, J. M., Marchant, J. L., \& Frith, C. D. (2006). Modulation of the mirror system by social relevance. Social Cognitive and Affective Neuroscience, 1(2), 143-148. doi:10.1093/scan/nsI017

Kilner, J. M., Neal, A., Weiskopf, N., Friston, K. J., \& Frith, C. D. (2009). Evidence of mirror neurons in human inferior frontal gyrus. The Journal of Neuroscience, 29(32), 1015310159. doi:10.1523/JNEUROSCI.2668-09.2009

Krings, T., Naujokat, C., Graf v. Keyserlingk, D. (1998). Representation of cortical motor function as revealed by stereotactic transcranial magnetic stimulation. Electroencephalography and Clinical Neurophysiology, 102(2), 85-93.

Kylliäinen, A., \& Hietanen, J. K. (2006). Skin conductance responses to another person's gaze in children with autism. Journal of Autism and Developmental Disorders, 36(4), 517-525. doi:10.1007/s10803-006-0091-4

Kylliäinen, A., Wallace, S., Coutanche, M. N., Leppänen, J. M., Cusack, J., Bailey, A. J., \& Hietanen, J. K. (2012). Affective-motivational brain responses to direct gaze in children with autism spectrum disorder. Journal of Child Psychology and Psychiatry, 53(7), 790797. doi:10.1111/j.1469-7610.2011.02522.x

Maeda, F., Chang, V. Y., Mazziotta, J., \& lacoboni, M. (2001). Experience-dependent modulation of motor corticospinal excitability during action observation. Experimental Brain Research, 140(2), 241-244. doi:10.1007/s002210100827

Maeda, F., Kleiner-Fisman, G., \& Pascual-Leone, A. (2002). Motor facilitation while observing hand actions: Specificity of the effect and role of observer's orientation. Journal of Neurophysiology, 87(3), 1329-1335.

Mahon, B. Z. (2015). What is embodied about cognition? Language, Cognition and Neuroscience, 30(4), 420-429. doi: 10.1080/23273798.2014.987791

Myllyneva, A., Ranta, K., \& Hietanen, J. K. (2015). Psychophysiological responses to eye contact in adolescents with social anxiety disorder. Biological Psychology, 109, 151-158. doi:10.1016/j.biopsycho.2015.05.005

Noens, I., De la Marche, W., \& Scholte, E. (2012). SRS-A - Screeningslijst voor autismespectrumstoornissen bij volwassenen. Handleiding [SRS-A - Screening tool for autism spectrum disorders in adults. A manual]. Amsterdam, The Netherlands: Hogrefe Uitgevers.

Nummenmaa, L., \& Calder, A. J. (2009). Neural mechanisms of social attention. Trends in Cognitive Sciences, 13(3), 135-143. doi:10.1016/j.tics.2008.12.006

Oberman, L. M., Hubbard, E. M., McCleery, J. P., Altschuler, E. L., Ramachandran, V. S., \& Pineda, J. A. (2005). EEG evidence for mirror neuron dysfunction in autism spectrum 
disorders. Brain

Research,

24(2),

190-198.

doi:10.1016/j.cogbrainres.2005.01.014

Obhi, S. S., Hogeveen, J., \& Pascual-Leone, A. (2011). Resonating with others: the effects of self-construal type on motor cortical output. The Journal of Neuroscience, 31(41), 1453114535. doi:10.1523/JNEUROSCI.3186-11.2011

Oldfield, R. C. (1971). The assessment and analysis of handedness: The Edinburgh inventory. Neuropsychologia, 9(1), 97-113. doi:10.1016/0028-3932(71)90067-4

Pageler, N. M., Menon, V., Merin, N. M., Eliez, S., Brown, W. E., \& Reiss, A. L. (2003). Effect of head orientation on gaze processing in fusiform gyrus and superior temporal sulcus. Neurolmage, 20(1), 318-329. doi:10.1016/S1053-8119(03)00229-5

Pelphrey, K. A., Viola, R. J., \& McCarthy, G. (2004). When strangers pass: Processing of mutual and averted social gaze in the superior temporal sulcus. Psychological Science, 15(9), 598-603. doi:10.1111/j.0956-7976.2004.00726.x

Perrett, D. I., Hietanen, J. K., Oram, M. W., \& Benson, P. J. (1992). Organization and functions of cells responsive to faces in the temporal cortex. Philosophical Transactions of the Royal Society of London. Series B, Biological Sciences, 335(1273), 23-30. doi:10.1098/rstb.1992.0003

Pönkänen, L. M., Peltola, M. J., \& Hietanen, J. K. (2011). The observer observed: frontal EEG asymmetry and autonomic responses differentiate between another person's direct and averted gaze when the face is seen live. International Journal of Psychophysiology, 82(2), 180-7. doi:10.1016/j.ijpsycho.2011.08.006

Ramachandran, V. S., \& Oberman, L. M. (2006). Broken mirrors: A theory of autism. Scientific American, 295(5), 62-69. doi:10.1038/scientificamerican1106-62

Rizzolatti, G., Camarda, R., Fogassi, L., Gentilucci, M., Luppino, G., \& Matelli, M. (1988). Functional organization of inferior area 6 in the macaque monkey. Experimental Brain Research, 71(3), 491-507. doi:10.1007/BF00248742

Rizzolatti, G., \& Craighero, L. (2004). The mirror neuron system. Annual Review of Neuroscience, 27, 169-192. doi:10.1146/annurev.neuro.27.070203.144230

Rizzolatti, G., \& Fabbri-Destro, M. (2008). The mirror system and its role in social cognition. Current Opinion in Neurobiology, 18(2), 179-184. doi:10.1016/j.conb.2008.08.001

Rizzolatti, G., Fadiga, L., Gallese, V., \& Fogassi, L. (1996). Premotor cortex and the recognition of motor actions. Cognitive Brain Research, 3(2), 131-141. doi:10.1016/09266410(95)00038-0

Rizzolatti, G., Fadiga, L., Matelli, M., Bettinardi, V., Paulesu, E., Perani, D., \& Fazio, F. (1996). Localization of grasp representations in humans by PET: 1. Observation versus execution. Experimental Brain Research, 111(2), 246-252. doi:10.1007/BF00227301

Rossini, P. M., Barker, A. T., Berardelli, A., Caramia, M. D., Caruso, G., Cracco, R. Q., ... 
Tomberg, C. (1994). Non-invasive electrical and magnetic stimulation of the brain, spinal cord and roots: basic principles and procedures for routine clinical application. Report of an IFCN committee. Electroencephalography and Clinical Neurophysiology, 91(2), 7992. doi:10.1016/0013-4694(94)90029-9

Scheiber, M. H. (1990). How might the motor cortex individuate movements. Trends in Neurosciences, 13, 440-445.

Senju, A., \& Johnson, M. H. (2009a). Atypical eye contact in autism: Models, mechanisms and development. Neuroscience and Biobehavioral Reviews, 33(8), 1204-1214. doi:10.1016/j.neubiorev.2009.06.001

Senju, A., \& Johnson, M. H. (2009b). The eye contact effect: Mechanisms and development. Trends in Cognitive Sciences, 13(3), 127-134. doi:10.1016/j.tics.2008.11.009

Strafella, A. P., \& Paus, T. (2000). Modulation of cortical excitability during action observation: A transcranial magnetic stimulation study. NeuroReport, 11(10), 2289-2292. doi:doi.org/10.1097/00001756-200007140-00044

Uusberg, H., Allik, J., \& Hietanen, J. (2015). Eye contact reveals a relationship between Neuroticism and anterior EEG asymmetry. Neuropsychologia, 76, 161-168.

Wang, Y., \& Hamilton, A. F. de C. (2012). Social top-down response modulation (STORM): a model of the control of mimicry in social interaction. Frontiers in Human Neuroscience, 6, 1-10. doi:10.3389/fnhum.2012.00153

Wang, Y., Newport, R., \& Hamilton, A. F. de C. (2011). Eye contact enhances mimicry of intransitive hand movements. Biology Letters, 7(1), 7-10. doi:10.1098/rsbl.2010.0279

Wang, Y., Ramsey, R., \& Hamilton, A. F. de C. (2011). The control of mimicry by eye contact is mediated by medial prefrontal cortex. Journal of Neuroscience, 31(33), 12001-12010. doi:10.1523/JNEUROSCI.0845-11.2011

Wieser, M. J., Pauli, P., Alpers, G. W., \& Mühlberger, A. (2009). Is eye to eye contact really threatening and avoided in social anxiety? An eye-tracking and psychophysiology study. Journal of Anxiety Disorders, 23(1), 93-103. doi:10.1016/j.janxdis.2008.04.004 


\section{Supplementary materials}

\section{A. Supplementary methods: measurement of muscle activity during movement execution}

Participants. Eight subjects (4 male, age range 18 - 24) participated in an additional 'movement execution' experiment measuring electromyography (EMG) during the actual execution of a hand opening movement. All participants were right-handed and provided written informed consents. Participants of this 'movement execution' experiment were not the same as those participating in the main 'movement observation' TMS experiment.

Task. In twelve trials, participants were instructed to observe and simultaneously execute the hand opening movement adopted in the main movement observation experiment. Additionally, twelve trials were recorded during which the hand was held static (not performing a movement).

EMG. During the execution of the movement, a surface electromyogram was simultaneously recorded from the APB and FDI hand muscles. EMG was quantified by calculating the root mean square error (RMSE) across an interval of 100 milliseconds which overlapped the time point of TMS stimulation in the main movement observation experiment (see main figure 1).

Data analysis. For each participant, EMG scores were averaged across trials, separately for each muscle and movement condition. A total of $13.02 \%$ of all trials were identified as outliers and were removed from the analysis (see main manuscript for outlier detection procedure). $A$ repeated measures ANOVA design on the EMG data was performed with the within-subjects factors 'muscle' (APB, FDI) and 'hand movement' (opening, static).

Results. The ANOVA analysis revealed a significant main effect of 'muscle' $(F(1,7)=31.99, p$ $<.001$ ), indicating significantly higher muscle activation for the APB compared to the FDI. Also a significant main effect of 'hand movement' $(F(1,7)=37.10, p<.001)$ was revealed, indicating significantly larger muscle activity during the execution of the hand opening movement, compared to static hand condition in both muscles. However, a significant 'muscle by hand movement' interaction $(F(1,7)=23.74, p<.001)$, indicated that the differential muscle activation was significantly more pronounced in the APB compared to the FDI. 


\section{B. Supplementary tables}

Table B.1.

Recorded background EMG data (RMSE-scores) separately for each muscle (APB, FDI) and condition. Data are represented as mean \pm standard deviation $(N=32)$.

\begin{tabular}{cccc}
\hline Muscle & Gaze & Movement & EMG $(\boldsymbol{\mu V})$ \\
\hline \multirow{3}{*}{ APB } & Averted & Opening & $0.0080 \pm 0.0095$ \\
& & Static & $0.0072 \pm 0.0066$ \\
\cline { 2 - 4 } & \multirow{2}{*}{ Direct } & Opening & $0.0079 \pm 0.0107$ \\
& \multirow{2}{*}{ Averted } & Static & $0.0076 \pm 0.0088$ \\
\hline \multirow{2}{*}{ FDI } & Opening & $0.0086 \pm 0.0076$ \\
& \multirow{2}{*}{ Direct } & Static & $0.0085 \pm 0.0085$ \\
& & Opening & $0.0084 \pm 0.0072$ \\
& & Static & $0.0084 \pm 0.0075$
\end{tabular}

Table B.2.

Linear regression beta-values for assessing the relationship between the SRS-A (subscale) scores and the 'gaze effect' (differential MEP response for direct versus averted gaze conditions) for observation of the hand opening movement (separately for the APB and FDI muscle) $(N=32)$.

\begin{tabular}{lcccccc}
\hline \multirow{2}{*}{ SRS-A subscale } & \multicolumn{3}{c}{ ABP } & & \multicolumn{2}{c}{ FDI } \\
\cline { 2 - 7 } & $\boldsymbol{\beta}$ & $\boldsymbol{t ( 3 0 )}$ & $\boldsymbol{p}$ & $\boldsymbol{\beta}$ & $\boldsymbol{t ( 3 0 )}$ & $\boldsymbol{p}$ \\
\hline Total score & -.10 & -0.56 & .57 & .11 & 0.62 & .54 \\
Social awareness & -.21 & -1.18 & .25 & .11 & 0.63 & .54 \\
Social communication & .04 & 0.22 & .83 & .18 & 0.99 & .33 \\
Social motivation & -.05 & -0.28 & .78 & .11 & 0.59 & .56 \\
Rigidity/repetitiveness & -.19 & -1.05 & .30 & -.08 & -0.46 & .65 \\
\hline
\end{tabular}




\section{Table B.3.}

Recorded gaze behavior (total fixation duration (TFD) and fixation count (FC)), separately for each dependent measure, area-of-interest (AOI: hand, eyes) and observation condition. Data are represented as mean \pm standard deviation $(N=28)$.

\begin{tabular}{lllll}
\hline AOI & Gaze & Movement & TFD & FC \\
\hline \multirow{3}{*}{ Hand } & Averted & Opening & $5.57 \pm 3.73$ & $12.64 \pm 7.89$ \\
& & Static & $4.37 \pm 3.92$ & $10.32 \pm 8.52$ \\
\cline { 2 - 5 } & \multirow{2}{*}{ Direct } & Opening & $5.85 \pm 4.35$ & $12.82 \pm 10.14$ \\
& Static & $3.37 \pm 3.30$ & $8.29 \pm 8.37$ \\
\hline \multirow{2}{*}{ Eyes } & Averted & Opening & $7.24 \pm 3.98$ & $13.68 \pm 7.18$ \\
& & Static & $9.38 \pm 5.15$ & $13.32 \pm 7.66$ \\
\cline { 2 - 5 } & \multirow{2}{*}{ Direct } & Opening & $9.81 \pm 6.14$ & $13.54 \pm 8.57$ \\
& & Static & $9.65 \pm 4.30$ & $13.46 \pm 7.04$ \\
\hline
\end{tabular}

